\title{
Proteção cerebral no tratamento cirúrgico dos aneurismas do arco aórtico: estudo experimental em cães
}

Gaudêncio Lopes ESPINOSA*, Henrique MURAD*, Antônio de Pádua JAZBIK*, Eduardo Sérgio BASTOS $^{\star}$, João de Deus e BRITO*, José L. FEITOSA*, Rogério Antônio Silva BARROS ${ }^{\star \star}$, Cláudio Miguel D'ÁVILA*

ESPINOSA, G. L.; MURAD, H.; JAZBIK, A. P.; BASTOS, E. S.; BRITO, J. D.; FEITOSA, J. L.; BARROS, R. A. S.; D'ÁVILA , C. M. - Proteção cerebral no tratamento cirúrgico dos aneurismas do arco aórtico: estudo experimental em cães. Rev. Bras. Cir. Cardiovasc., 9 (4): 193-204, 1994.

RESUMO: É realizado estudo experimental comparativo entre dois métodos de proteção cerebral na abordagem cirúrgica dos aneurismas do arco aórtico, avaliando a sua eficácia: hipotermia sistêmica profunda isolada (menor que $20^{\circ} \mathrm{C}$ ) com pinçamento arterial braquiocefálico e hipotermia sistêmica profunda associada à perfusão carotídea seletiva. Dois grupos de 15 cães cada foram submetidos, respectivamente, à hipotermia sistêmica profunda com pinçamento arterial braquiocefálico (Grupo I) e à hipotermia sistêmica profunda associada à perfusão seletiva da carótida direita (Grupo II). Foram analisadas amostras seriadas das alteraçōes metabólicas de $\mathrm{pH}$ e $\mathrm{PaCO}_{2}$ que ocorreram no retorno venoso cerebral aferidas na veia julgular interna, bem como as alterações histopatológicas encontradas com $45 \mathrm{~min}$., $90 \mathrm{~min}$. e $135 \mathrm{~min}$. de cada procedimento. Os resultados demonstram que, apesar de ambos os métodos de proteção cerebral serem eficazes por um período de 45 minutos, o método utilizado no Grupo II mostrou ser superior em períodos de até 90 minutos de isquemia cerebral. Em períodos de 135 minutos os métodos tiveram resultados semelhantes, não oferecendo proteção cerebral adequada.

DESCRITORES: proteção cerebral; aneurismas do arco aórtico, cirurgia.

\section{INTRODUÇÃO}

Os aneurismas localizados no arco aórtico constituem uma grave forma de doença, tendo o seu tratamento se convertido em um desafio para os cirurgiōes cardiovasculares.

O arco aórtico é definido como o segmento da aorta onde se originam as artérias braquiocefálicas. Estende-se desde a origem do tronco arterial braquiocefálico até o segmento aórtico, logo após a emergência da artéria subclávia esquerda.

Uma vez realizado o diagnóstico, o tratamento cirúrgico está indicado em todos os casos em que não exista associada uma condição mórbida grave sem perspectivas terapêticas. O tratamento é realizado mediante reconstituição com prótese vascular e reimplante dos três grandes vasos.

As principais dificuldades incluem a proteção do tecido cerebral e da medula espinhal de lesões isquêmicas ou embólicas, prevenção de coagulopatias e de lesões do músculo cardíaco durante a circulação extracorpórea (CEC).

Apesar das complicações neurológicas terem diminuído bastante nos últimos anos graças aos progressos das técnicas e equipamentos cirúrgicos, ainda não se tornaram um fato incomum. A neces-

Trabalho realizado no Serviço de Cirurgia Cardiaca do Universitário Clementino Fraga Filho da Universidade Federal do Rio de Janeiro. Rio de Janeiro, RJ, Brasil.

Recebido para publicação em 10 de março, 1994.

" Do Hospital Universitário Clementino Fraga Filho.

** Do Hospital Miguel Couto.

Endereço para correspondência: Gaudêncio Espinosa. Praia de Icaraí, 113, apt 801 . Niterói, Rio de Janeiro, RJ, Brasil. 
ESPINOSA, G. L.; MURAD, H.; JAZBIK, A. P.; BASTOS, E. S.; BRITO, J. D.; FEITOSA, J. L.; BARROS, R. A. S.; D'ÁVILA, C. M. - Proteção cerebral no tratamento cirúrgico dos aneurismas do arco aórtico: estudo experimental em cães. Rev. Bras. Cir. Cardiovasc., 9 (4): 193-204, 1994.

sidade do pinçamento arterial proximal na aorta ascendente e distal logo após os vasos braquiocefálicos, aumenta o risco de dano cerebral por esquêmia.

Várias técnicas têm sido empregadas para proteção do tecido nervoso, tais como o uso temporário de derivações com tubos ${ }^{6}$, o uso de enxertos temporários ou definitivos 25 , perfusão das artérias braquiocefálicas ${ }^{21}$ ou o uso de circulação extracorpórea e hipotermia profunda ${ }^{16} \mathrm{com}$ ou sem parada cardiocirculatória total. $\mathrm{O}$ risco de dano cerebral no entanto, devido a proteção inadequada, tempo cirúrgico prolongado, embolias ou lesão da microcirculação, ainda existe.

Atualmente, a técnica cirúrgica utilizada mais freqüentemente com poucas modificações é a descrita por GRIEPP et alii ${ }^{16}$, em 1975 , que consiste em: circulação estracorpórea, hipotermia profunda e parada cardiocirculatória total. Em 1987, LUOSTO et alii ${ }^{21}$ propuzeram um método complementar a esta técnica consistindo na colocação de cânula na carotída para perfusão seletiva a partir da linha arterial da bomba de CEC efetuando-se perfusão cerebral carotídea com sangue frio durante o tempo cirúrgico principal em que os vasos arteriais braquiocefálicos encontram-se pinçados. Esta última técnica é bastante promissora e parece conferir uma proteção cerebral adequada durante a operação. A experiência é muito restrita, por se tratar de método recente.

Baseados nos estudos de GRIEPP et alii ${ }^{16} \mathrm{e}$ LUOSTO et alii ${ }^{21}$, decidiu-se realizar um trabalho experimental em cães, visando avaliar o risco do dano cerebral que pode ocorrer na técnica preconizada por esses autores. Utilizou-se para tal, as alterações do $\mathrm{pH}$ e $\mathrm{PaCO}_{2}$ sangüíneas que ocorrem na veia jugular interna durante cada um desses procedimentos, bem como a histopatologia do sistema nervoso central dos animais após cada operação, com o objetivo de localizar possíveis lesões anatomopatológicas.

\section{CASUÍSTICA E MÉTODOS}

Dois grupos de 15 cães mestiços - num total de 30 cães - pesando entre $7,1 \mathrm{~kg}$ e $18,2 \mathrm{~kg}$ (média de $12,4 \mathrm{~kg}$ ), foram submetidos a duas técnicas cirúrgicas diferentes visando compará-las quanto ao grau de proteção cerebral.

O primeiro grupo de 15 cães (Grupo I), pesando entre $7,1 \mathrm{~kg}$ e $17,7 \mathrm{~kg}$ (média de $12,1 \mathrm{~kg}$ ), foi submetido a CEC e resfriamento sistêmico até a temperatura corporal de $18^{\circ} \mathrm{C} / 20^{\circ} \mathrm{C}$, quando então pinçou-se o tronco arterial braquiocefálico (no cão as artérias subclávia direita, carótida esquerda e carótida direita originam-se de um único tronco arterial braquiocefálico) e a artéria subclávia esquerda, impedindo assim qualquer fluxo sangūíneo cerebral, quer pelas carótidas quer pelas artérias vertebrais. O tempo de pinçamento arterial foi de 45 minutos para 5 cães, de 90 minutos para outros 5 cães e de 135 minutos para os 5 restantes.

O segundo grupo (Grupo II), igualmente composto de 15 cães, pesando entre $7,8 \mathrm{~kg}$ e $18 \mathrm{~kg}$ (média de 13,2 kg), foi submetido da mesma forma que $\circ$ grupo anterior a CEC, resfriamento sistêmico $\left(18^{\circ} \mathrm{C} / 20^{\circ} \mathrm{C}\right)$ e pinçamento dos vasos arteriais braquiocefálicos, sendo porém introduzida cânula na carótida direita para perfusão seletiva, a partir da linha arterial proveniente da bomba de CEC mantendo-se, com isto, um fluxo sangüíneo cerebral durante o tempo de pinçamento arterial. Os tempos de pinçamento arterial foram idênticos aos efetuados no primeiro grupo (Figura 1).

Antes da realização do procedimento cirúrgico, os animais foram submetidos a um tempo de observação mínimo de três semanas e exame veterinário minucioso.

Os animais foram sedados com administração intramuscular de Ketamina $(5 \mathrm{mg} / \mathrm{kg})$ e atropina $(0,25 \mathrm{mg})$. $\mathrm{Na}$ indução anestésica utilizou-se tiopental sódico na dose de $4 \mathrm{mg} / \mathrm{kg} /$ dose, sendo intubados e submetidos à ventilação mecânica através de respirador (modelo NSH-34RH Harvard Apparatus) com manutenção da narcose geral por inalação de metoxiflurano.

Feita dissecção da artéria femoral esquerda, com dispositivo para medida contínua da pressão arterial média. A seguir, realizou-se a dissecção de artéria e veia femoral direitas. Na veia foi colocada cânula como via de hidratação e administração de medicamentos, e a artéria femoral para posterior utilização como via de retorno arterial proveniente da bomba de circulação extracorpórea.

Após assepsia e antissepsia, realizou-se toracotomia mediana com abertura do saco pericárdico e sutura em bolsa na aurícula direita com colocação de cânula no átrio, após heparinização sistêmica com $200 \mathrm{u} / \mathrm{kg}$ de heparina sódica.

Instituída, a seguir, a circulação extracorpórea com fluxo de $60 \mathrm{ml} / \mathrm{kg} / \mathrm{min}$., utilizando-se bomba (modelo IC3 do Instituto Dante Pazzanese de Cardiologia ) com seu respectivo oxigenador pediátrico reutilizável. A pressão arterial média foi mantida em torno de $60 \mathrm{mmHg}$.

O circuito de CEC, bem como o oxigenador foram previamente preenchidos com solução de Ringer com lactato $500 \mathrm{ml}$, manitol $20 \mathrm{ml}$ e hidrocortisona $150 \mathrm{mg}$ e a oxigenação sangüínea durante a CEC foi com fluxo constante de oxigênio a $100 \%$, não sendo adicionado dióxido de carbono. 


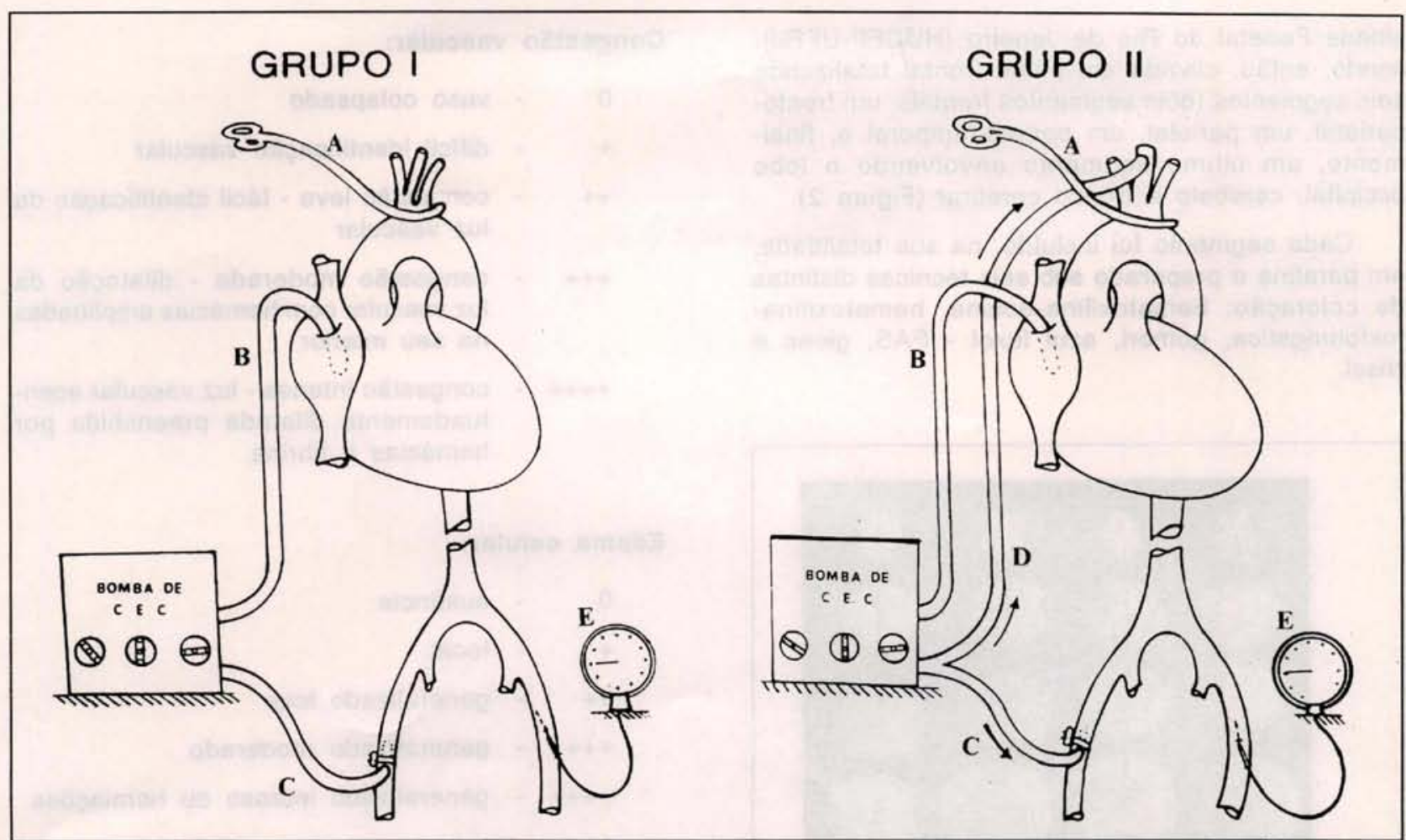

Fig. 1 - Esquema comparativo da técnica cirúrgica empregada no Grupo I e no Grupo II. A: pinçamento dos vasos arteriais braquiocefálicos. B: drenagem venosa do átrio direito. C: linha arterial proveniente da bomba de CEC instalada em artéria femoral direita. D: linha arterial utilizada na perfusão carotidea (Grupo II) a partir da linha femoral. E: monotorização contínua da pressão média em artéria femoral esquerda.

Durante o resfriamento sistêmico até uma temperatura de $18^{\circ} \mathrm{C} / 20^{\circ} \mathrm{C}$, realizou-se a dissecção do arco aórtico, com identificação e reparo do tronco arterial braquiocefálico e da artéria subclávia esquerda. Posteriormente, dissecou-se a veia jugular interna direita e colocada cânula em direção cefálica até as proximidades do seio cavernoso.

Uma vez atingida a temperatura corporal de $18^{\circ} \mathrm{C} / 20^{\circ} \mathrm{C}$, aferida através de teletermômetro (Yellow Springs-Ohio), cujo sensor foi inserido por via retal, efetuou-se o pinçamento dos vasos braquiocefálicos, impedindo, dessa forma, o fluxo sangüíneo cerebral. Nos animais do Grupo II realizou-se, previamente ao pinçamento arterial, a colocação de cânula na carótida direita, mantendo, com isto, o fluxo sangüíneo cerebral durante o período de pinçamento, com sangue a $18^{\circ} \mathrm{C} / 20^{\circ} \mathrm{C}$ proveniente da linha arterial da bomba de CEC.

Durante o tempo de pinçamento arterial, a CEC foi mantida como método de proteção medular, bem como dos demais órgãos vitais. A linha arterial da CEC também serviu como via de perfusão cerebral seletiva nos animais em que se efetuou a perfusão carotídea.

Logo após, foram colhidas amostras sangũineas venosas de $15 \mathrm{~min}$. em $15 \mathrm{~min}$. através do cateter inserido na veia jugular interna para realização de gasometrias venosas seriadas, aferidas através do ph/Blood Gas 16512 Corning, equipamento específico para medida de gases sangüíneos. Os dados obtidos foram submetidos ao teste $T$ de Student, para avaliar a significância de amostras isoladas.

Após o período de pinçamento, o cão foi reaquecido até a temperatura sistêmica de $35^{\circ} \mathrm{C}$, na qual foi mantido durante um período de $\mathbf{3 0}$ minutos, após o que foi sacrificado.

Em seguida, realizou-se perfusão carotídea bilateral com $150 \mathrm{ml}$ de solução de Cajal ${ }^{*}$, após o que efetuou-se trepanação com retirada das massas cerebral, cerebelar e do tronco cerebral, sendo submersas em solução de Cajal, evitando retração das estruturas e proporcionando melhor conservação do tecido para posterior análise histopatológica.

A massa encefálica, pesando entre 50 e $90 \mathrm{~g}$, foi encaminhada ao Serviço de Patologia do Hospital Universitário Clementino Fraga Filho da Univer-

\footnotetext{
* Solução de Cajal: Fixador utilizado para tecido nervoso com tempo de fixação de 2 a 15 dias.

Composição por $100 \mathrm{ml}$ : Aldeído fórmico (37 a 40\%) $15 \mathrm{ml}$ Brometo de amônio $\quad 2 \mathrm{~g}$ Água destilada $\quad 85 \mathrm{ml}$
} 
ESPINOSA, G. L.; MURAD, H.; JAZBIK, A. P.; BASTOS, E. S.; BRITO, J. D.; FEITOSA, J. L.; BARROS, R. A. S.; D'ÁVILA, C. M. - Proteção cerebral no tratamento cirúrgico dos aneurismas do arco aórtico: estudo experimental em cães. Rev. Bras. Cir. Cardiovasc., 9 (4): 193-204, 1994.

sidade Federal do Rio de Janeiro (HUCFF-UFRJ), sendo, então, clivada em plano frontal totalizando seis segmentos (dois segmentos frontais, um frontoparietal, um parietal, um parieto-temporal e, finalmente, um último segmento envolvendo o lobo occipital, cerebelo e tronco cerebral (Figura 2).

Cada segmento foi incluído, na sua totalidade, em parafina e preparado sob seis técnicas distintas de coloração: hematoxilina-eosina, hematoxilinafosfotungstica, gomori, azul luxol - PAS, glees e Nissl.

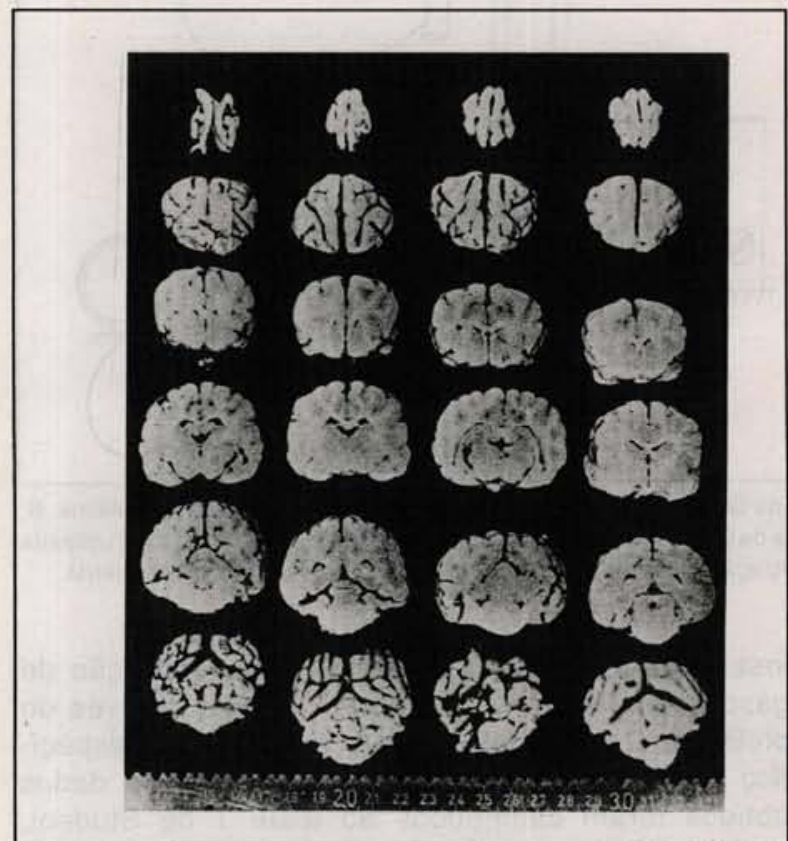

Fig. 2 - Visão dos diversos segmentos encefálicos nos quais realizou-se análise histopatológica. De cima para baixo: dois frontais, um fronto-parietal, um parietal, um parieto-temporal e um último segmento envolvendo o lobo occipital, o cerebelo e o tronco cerebral.

A intensidade das lesões foi classificada segundo os critérios a seguir:

Isquemia celular neuronal:

$$
\begin{aligned}
& 0 \text { - ausência } \\
& +\quad-\text { unifocal } \\
& \text { ++ - multifocal leve - raros neurônios } \\
& \text { isquêmicos } \\
& \text { +++ - multifocal moderada - numerosos } \\
& \text { neurônios isquêmicos } \\
& \text { ++++ - multifocal intensa ou confluente - } \\
& \text { marcantes alterações celulares com } \\
& \text { tendência a lesão difusa ou laminar }
\end{aligned}
$$

\section{Congestão vascular:}

$$
\begin{aligned}
& 0 \text { - vaso colapsado } \\
& \text { + - difícil identificação vascular } \\
& \text { ++ - congestão leve - fácil identificação da }
\end{aligned}
$$

\section{Edema celular:}

$$
\begin{array}{ll}
0 & - \text { ausência } \\
+ & - \text { focal } \\
++ & - \text { generalizado leve } \\
+++ & - \text { generalizado moderado } \\
++++ & - \text { generalizado intenso ou herniações }
\end{array}
$$

Analisando o conjunto das alterações histopatológicas e considerando como mais significativas as alterações neuronais, graduamos as lesões de cada amostra em ausentes, leves, moderadas, severas e muito severas.

\section{RESULTADOS}

\section{1) Alterações Metabólicas ( $\mathrm{pH}$ e $\mathrm{PaCO}_{2}$ )}

A variação do $\mathrm{pH}$ sangüíneo, aferido na veia jugular interna nos cães do Grupo I durante o tempo de pinçamento arterial em vigência de hipotermia profunda, iniciou-se com um valor médio de 7,04 e, com o decorrer do tempo, foi se tornando mais ácido. A partir dos 45 minutos, esta queda tornouse muito acelerada e, aos 135 minutos - ao final do tempo de pinçamento -, o valor médio era de 6,28. Desta forma, podemos observar que, no decorrer do tempo de pinçamento, ocorreu uma acidificação sangüínea que se tornou mais intensa a partir dos 45 minutos.

A variação da $\mathrm{PaCO}_{2}$ sangüínea neste mesmo grupo de cães (avaliada da mesma forma como o $\mathrm{pH}$ ) iniciou-se em um valor médio de $40,8 \mathrm{mmHg}$ que foi se tornando maior com o decorrer do tempo.

Coincidentemente com as variações do $\mathrm{pH}$, as alterações da $\mathrm{PaCO}_{2}$ a partir dos 45 minutos tornaram-se significantes e, aos 135 minutos - final do 
ESPINOSA, G. L.; MURAD, H.; JAZBIK, A. P.; BASTOS, E. S.; BRITO, J. D.; FEITOSA, J. L.; BARROS, R. A. S.; D'ÁVILA, C. M. - Proteção cerebral no tratamento cirúrgico dos aneurismas do arco aórtico: estudo experimental em cães. Rev. Bras. Cir. Cardiovasc., 9 (4): 193-204, 1994.

pinçamento - o valor médio encontrado era de 143,8 $\mathrm{mmHg}$. Desta forma, podemos observar que, com o decorrer do tempo de pinçamento, ocorreu uma hipercapnia mais intensa a partir dos 45 minutos.

Quando comparamos as variações de $\mathrm{pH}$ e $\mathrm{PaCO}_{2}$ do Grupo I entre si, notamos nitidamente que, a partir dos 45 minutos, estas alteraçōes tornam-se muito mais intensas.

Quando observamos a variação do $\mathrm{pH}$ sangüíneo aferido na veia jugular interna nos cães do Grupo II, observamos que ocorre uma acidificação sangüínea no decorrer do tempo. O valor médio inicial de 7,15 atingiu, ao final do experimento, um valor de 6,54 . No entanto, a variação da $\mathrm{PaCO}_{2}$ não foi significante durante os 135 minutos do procedimento, iniciando-se em um valor médio de 38,3 $\mathrm{mmHg}$ e finalizando em $37,2 \mathrm{mmHg}$.

Quando comparamos as variações do $\mathrm{pH}$ dos dois grupos entre si (Tabela 1), notamos que a variação do $\mathrm{pH}$ do Grupo II foi menor que a do Grupo I. Esta diferença mostrou-se significativa $(p<0,05)$ a partir dos 45 minutos do procedimento.

A comparação das variaçōes da $\mathrm{PaCO}_{2}$ entre si (Tabela 2) demonstra que a variação no Grupo I foi muito grande, enquanto que a variação no Grupo II praticamente inexistiu, mantendo-se os valores médios estáveis durante os 135 minutos do procedimento. A diferença entre os grupos torna-se significativa $(p<0,05)$ a partir dos 30 minutos do procedimento.

\section{2) Alterações Histopatológicas}

Em cinco animais previamente estudados (con- trole) prevaleceu o padrão de normalidade neuronal, com células de contornos bem definidos, membrana citoplasmática razoavelmente nítida, membrana nuclear, nucléolos evidentes, cromatina frouxa e substância cromática de Nissl bem evidenciada.

Os vasos mostraram-se preenchidos por sangue e fibrina.

Dois animais desse grupo apresentaram raros neurônios corticais retraídos e acidófilos com borramento nuclear (expressão de agressão neuronal) e vasos relativamente dilatados. Nos demais aspectos, prevaleceu o padrão de normalidade no que se refere tanto à celularidade quanto às características morfológicas de neurônios, células de glia (astrócitos, oligodendrócitos e células ventriculares), dos vasos e das meninges.

\subsection{Grupo I}

Ao exame macroscópio, não houve herniação ou sofrimento hemorrágico em nenhum animal.

Ao exame microscópico, foi observada congestão vascular intensa predominantemente na região cortical, apresentando-se em menor grau nos núcleos da base e no tálamo, sendo pouco evidente na substância branca.

O edema celular esteve sempre presente em intensidade leve e moderada.

$\mathrm{A}$ isquemia celular caracterizou-se por marcante eosinofilia do citoplasma, perda da substância de Nissl e picnose nuclear em grande número de neurônios. Esta isquemia neuronal foi sempre mais

TABELA 1

COMPARAÇĀO DAS VARIAÇŌES DO PH ENTRE O GRUPO I E O GRUPO II, COM AS SUAS RESPECTIVAS SIGNIFICÂNCIAS SIGNIFICÂNCIA DA VARIAÇĀO DO pH

\begin{tabular}{|c|c|c|c|c|c|}
\hline & \multicolumn{2}{|c|}{ GRUPO I } & \multicolumn{3}{|c|}{ GRUPO II } \\
\hline & MÉDIA & $E P$ & MÉDIA & $E P$ & $P$ \\
\hline \multicolumn{6}{|l|}{ TEMPO (MIN.) } \\
\hline & 7,036 & 0,033 & 7,146 & 0,037 & 0,034 \\
\hline 15 & 7,025 & 0,023 & 7,060 & 0,020 & 0,251 \\
\hline 30 & 6,960 & 0,032 & 7,097 & 0,064 & 0,066 \\
\hline 45 & 6,855 & 0,030 & 6,935 & 0,020 & 0,034 \\
\hline 60 & 6,733 & 0,026 & 6,843 & 0,029 & 0,010 \\
\hline 75 & 6,769 & 0,022 & 6,856 & 0,023 & 0,013 \\
\hline 90 & 6,582 & 0,027 & 6,767 & 0,024 & $<0,001$ \\
\hline 105 & 6,426 & 0,046 & 6,630 & 0,069 & 0,039 \\
\hline 120 & 6,354 & 0,032 & 6,574 & 0,048 & 0,005 \\
\hline 135 & 6,280 & 0,040 & 6,540 & 0,035 & 0,001 \\
\hline
\end{tabular}

$E P=$ erro padrão

$P=$ significância da diferença entre as médias $(p<0,05)$. 
ESPINOSA, G. L.; MURAD, H.; JAZBIK, A. P.; BASTOS, E. S.; BRITO, J. D.; FEITOSA, J. L.; BARROS, R. A. S.; D'ÁVILA, C. M. - Proteção cerebral no tratamento cirúrgico dos aneurismas do arco aórtico: estudo experimental em cães. Rev. Bras. Cir. Cardiovasc., 9 (4): 193-204, 1994.

TABELA 2 COMPARAÇÃO DAS VARIAÇŌES DA PaCO2 ENTRE O GRUPOIE O GRUPO II, COM SUAS RESPECTIVAS SIGNIFICÂNCIAS SIGNIFICÂNCIA DA VARIAÇĀO DA PaCO

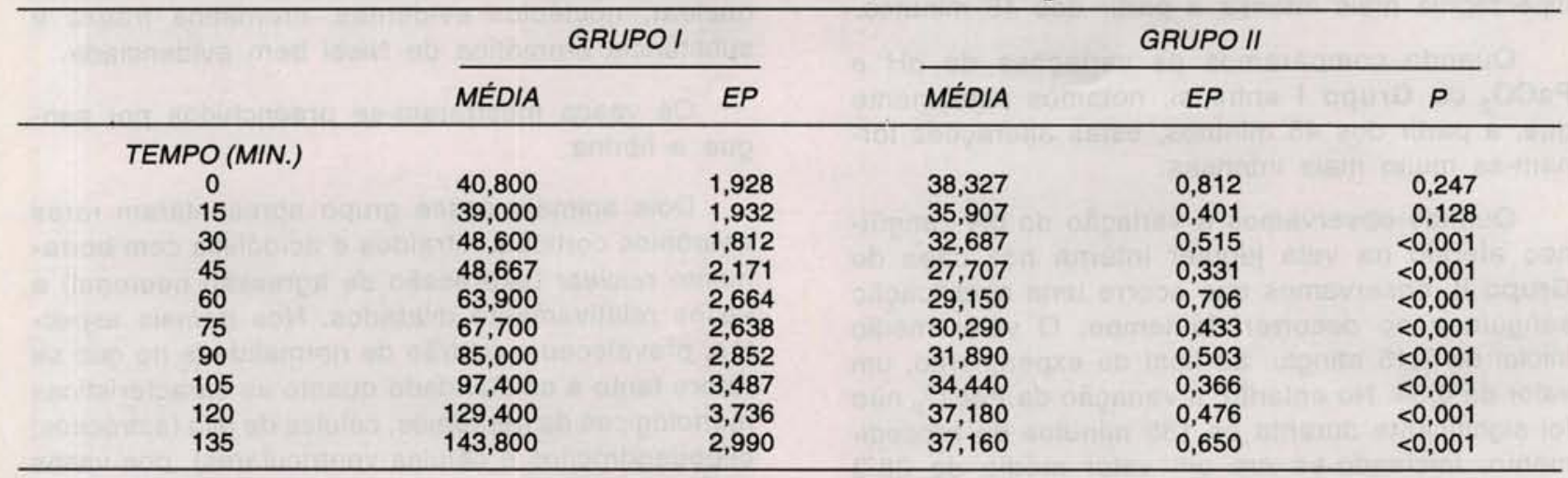

EP $=$ erro padrão

$P=$ significância da diferença entre as médias $(p<0,05)$.

intensa no córtex do que nos núcleos da base, tálamo e hipocampo (Figura 3).

A lesão cortical foi mais intensa e freqüente nos córtices frontal e occipital, na convexidade e na região medial. Os córtices parietal e temporal também exibiram comprometimento, porém, usualmente, menos intenso. As terceira, quarta e quinta camadas corticais foram as mais lesadas. As lesões foram evidenciadas igualmente no hemicórtex direito, bem como no esquerdo, não existindo diferenças de intensidade ou freqüência.

Nos animais submetidos a maior tempo de pinçamento arterial, ocorreram lesões neuronais isquêmicas mais amplas em maior número de focos, apresentando-se algumas vezes em lesão laminar ou com confluência tendendo a lesão difusa em áreas do córtex frontal ou occipital.

A lesão isquêmica da camada de Purkinge foi um achado constante. Variou muito em intensidade desde comprometimentos celulares isolados até grandes comprometimentos com lesão de várias áreas vizinhas, como foi observado nos animais submetidos a maior tempo de pinçamento arterial (Figura 4).

Não foram observadas alterações de mielina.

\subsection{Grupo II}

Ao exame macroscópico, dois animais apresentaram foco hemorrágico subaracnóideo. Não houve herniações.

Ao exame microscópico, foi observada congestão vascular intensa, predominante na região cortical, apresentando-se em menor grau nos núcleos da

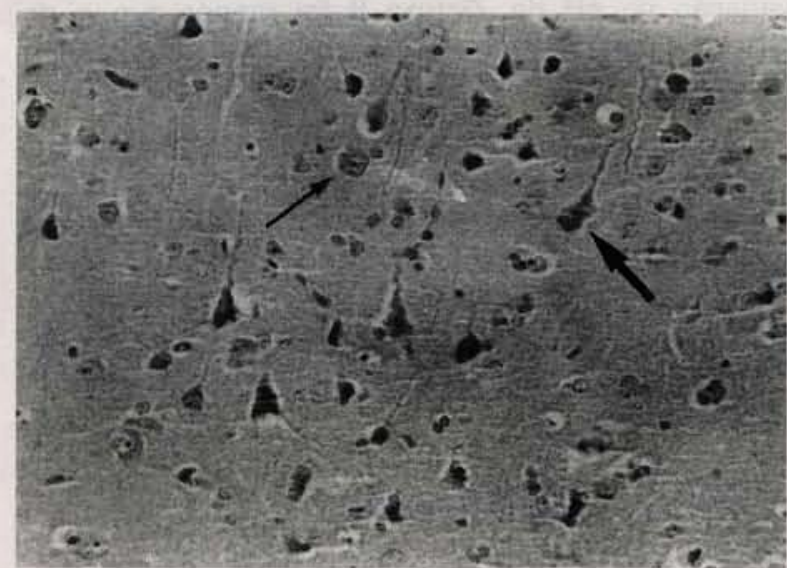

Fig. 3 - Córtex motor. Células piramidais com graus variados de alterações isquêmicas, que vão desde acentuada eosinofilia e retração de dentrito apical (seta larga) até retração e condensação de citoplasma e núcleo. Alguns neurônios piramidais normais (seta fina). Hematoxilina eosina: $40 \times 12,5$.

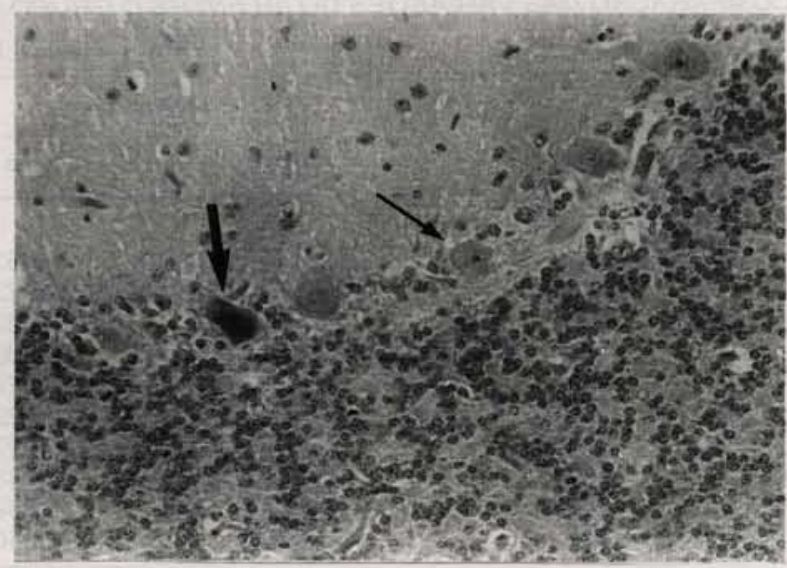

Fig. 4 - Córtex cerebelar. Célula de Purkinge normal (seta fina) ao lado de célula de Purkinge retraída e condensada (seta larga). Hematoxilina eosina: $40 \times 12,5$. 
ESPINOSA, G. L.; MURAD, H.; JAZBIK, A. P.; BASTOS, E. S.; BRITO, J. D.; FEITOSA, J. L.; BARROS, R. A. S.; D'ÁVILA, C. M. - Proteção cerebral no tratamento cirúrgico dos aneurismas do arco aórtico: estudo experimental em cães. Rev. Bras. Cir. Cardiovasc., 9 (4): 193-204, 1994.

base e tálamo, sendo pouco evidente na substância branca.

$\mathrm{Na}$ grande maioria dos casos, existiram artefatos com imagem negativa perivascular em substância branca-córtex, dando a impressão de grande edema.

A isquemia celular observada caracterizou-se por marcante eosinofilia do citoplasma, perda da substância de Nissl e picnose nuclear em grande número de neurônios. Esta isquemia neuronal foi sempre mais intensa no córtex do que nos núcleos da base, tálamo e hipocampo.

A lesão cortical foi mais intensa e freqüente no córtex frontal e occipital, na convexidade e na região medial. Os córtices parietal e temporal também exibiram comprometimento, porém, menos intenso. A terceira, quarta e quinta camadas corticais foram as mais lesadas. As lesões foram evidenciadas igualmente no hemicórtex direito bem como no esquerdo, não existindo diferenças de intensidade ou freqüência.

Lesões neuronais isquêmicas foram observadas na camada de Purkinge e, em menor número, nos núcleos cerebelares, principalmente no núcleo denteado.

A Tabela 3 mostra o grau de lesão estrutural cerebral no Grupo I (parada circulatória total) e no Grupo II (perfusão carotídea seletiva) aos $45 \mathrm{~min}$., $90 \mathrm{~min}$. e $135 \mathrm{~min}$.

\section{COMENTÁRIOS}

A primeira dentre as técnicas utilizadas para proteção cerebral, que consistia em perfusão dos vasos braquiocefálicos durante CEC com normotermia - proposta por DE BAKEY ${ }^{5}$ e COOLEY et alii $^{3}$ - associou-se a um alto número de complicações neurológicas. Com a evolução das técnicas de proteção cerebral, chegou-se, finalmente, à hipotermia profunda e parada cardiocirculatória total, técnica preconizada pela primeira vez por GRIEPP et alii ${ }^{16}$ e utilizada largamente até os dias atuais.

Apesar da hipotermia profunda com ou sem parada cardiocirculatória total ter provado ser um método de proteção cerebral eficiente, a ausência de fluxo sangüíneo para o sistema nervoso central confere um tempo de proteção limitado, o que pode acarretar dano neurológico em operações complexas ou reparos difíceis em que o tempo cirúrgico necessita ser prolongado.

Aparentemente, uma hora de ausência de fluxo sangüíneo cerebral durante hipotermia profunda $\left(20^{\circ} \mathrm{C}\right)$ tem sido considerado um tempo adequado.
TABELA 3

ALTERAÇÕES HISTOPATOLÓGICAS ENCONTRADAS NOS GRUPOS I (INTERRUPCÃO DA CIRCULAÇÃO CEREBRAL) E II (PERFUSĀO CAROTÍDEA SELETIVA)

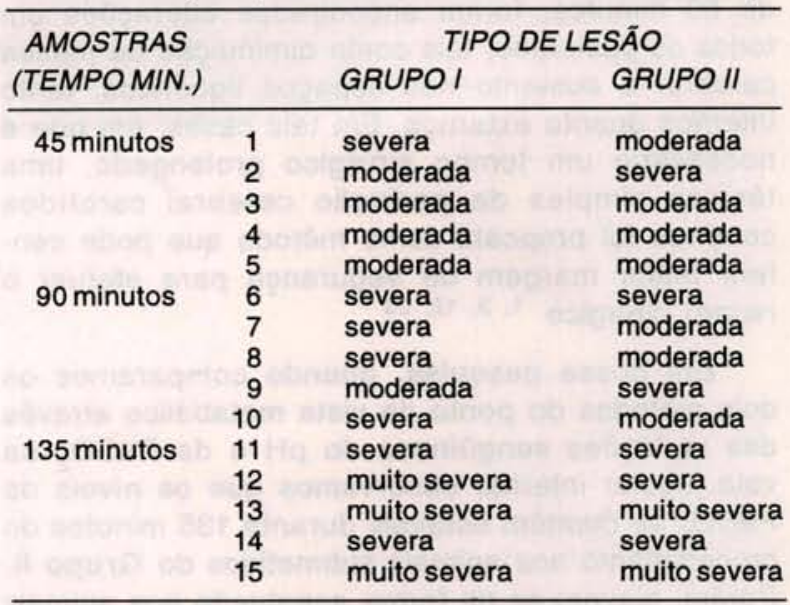

Porém, em nossa pesquisa, a hipotermia profunda a $18^{\circ} \mathrm{C} / 20^{\circ} \mathrm{C}$, seguida de pinçamento arterial dos vasos baquiocefálicos, conseguiu manter os processos metabólicos em níveis estáveis por um período de 45 minutos, após o qual observamos uma queda acentuada do $\mathrm{pH}$ e um aumento progressivo da $\mathrm{PaCO}_{2}$ na veia jugular interna, traduzindo importantes alterações metabólicas locais; isso sugere a possibilidade de que possa ocorrer dano cerebral após este período, confirmando estudos de outros autores ${ }^{29}$. Estas alterações devem-se provavelmente ao fato de que a hipotermia profunda não elimina o metabolismo cerebral, embora consiga reduzi-lo significativamente.

Estudos prévios sobre o metabolismo cerebral em cães durante hipotermia profunda $\left(20^{\circ} \mathrm{C}\right)$ concluíram que o consumo de oxigênio por parte do tecido cerebral, durante este tipo de procedimento, cai para níveis em torno de $15 \%$ a $30 \%$ em relação ao estado normotérmico ${ }^{27}$. Outros trabalhos em que, durante hipotermia profunda $\left(20^{\circ} \mathrm{C}\right)$, o consumo de oxigênio e o fluxo sangüíneo cerebral foram relacionados com a velocidade de perfusão sistêmica concluem que - apesar da hipotermia - continua ocorrendo consumo de oxigênio por parte do tecido cerebral ${ }^{10}$. Desta forma, quando a circulação cerebral encontra-se excluída da CEC, continuará existindo algum nível de processo metabólico tecidual, apesar da hipotermia profunda. Este fato, associado à ausência de fluxo cerebral que ocorre nestes procedimentos, propicia um acúmulo progressivo de catabólitos e substâncias tóxicas que, com o decorrer do tempo, acabam por lesar as estruturas celulares nervosas. 
ESPINOSA, G. L.; MURAD, H.; JAZBIK, A. P.; BASTOS, E. S.; BRITO, J. D.; FEITOSA, J. L.; BARROS, R. A. S.; D'ÁVILA, C. M. - Proteção cerebral no tratamento cirúrgico dos aneurismas do arco aórtico: estudo experimental em cães. Rev. Bras. Cir. Cardiovasc., 9 (4): 193-204, 1994.

Em estudos clínicos realizados através de tomografias computadorizada de crânio em pacientes submetidos a parada cardiocirculatória por mais de 60 minutos, foram encontradas alterações em todos os pacientes, tais como diminuição da massa cerebral e aumento nos espaços liquóricos, tanto internos quanto externos. Em tais casos, em que é necessário um tempo cirúrgico prolongado, uma técnica simples de perfusão cerebral carotídea contínua foi proposta como método que pode conferir maior margem de segurança para efetuar o reparo cirúrgico $1,3,12,23$.

Em nossa pesquisa, quando comparamos os dois métodos do ponto de vista metabólico através das variações sangüíneas do $\mathrm{pH}$ e da $\mathrm{PaCO}_{2}$ na veia jugular interna, observamos que os níveis da $\mathrm{PaCO}_{2}$ se mantêm estáveis durante 135 minutos do procedimento nos animais submetidos do Grupo II. Porém, elevam-se de forma acentuada nos animais em que apenas o pinçamento arterial foi efetuado. Este acúmulo progressivo de $\mathrm{CO}_{2}$ neste tipo de procedimento reflete de modo indireto um aumento gradativo dos metabólitos sangüíneos na circulação cerebral. No entanto, quando a perfusão carotídea é efetuada, os níveis de $\mathrm{PaCO}_{2}$, sangüíneos mantêm-se estáveis, levando-nos a crer que, de alguma forma, a manutenção do fluxo sangũíneo cerebral evita 0 acúmulo de metabólitos que podem ser lesivos ao tecido nervoso.

Quando estudamos as variações do $\mathrm{pH}$, também observamos melhores resultados com o uso da perfusão carotídea, porém não tão marcantes quanto no estudo das variações da $\mathrm{PaCO}_{2}$.

No grupo de animais com perfusão carotídea, notamos uma variação menor do $\mathrm{pH}$ que no grupo em que se utilizou apenas pinçamento arterial, quando a acidificação sangüínea foi marcante. No entanto, apesar da perfusão carotídea apresentar resultados mais satisfatórios, os níveis do $\mathrm{pH}$ não conseguiram manter-se tão estáveis quanto os níveis da $\mathrm{PaCO}_{2}$. Isto, provavelmente, deve-se a uma acidose metabólica progressiva que ocorre durante a CEC ${ }^{26}$ já que não foram efetuadas correções dos resultados gasométricos com substâncias alcalinas, a fim de não interferir com os resultados metabólicos aferidos.

O emprego das alterações metabólicas sangüineas que ocorreram na veia jugular interna como método de avaliação dos processos metabólicos intracerebrais, foi por nós utilizado com base na experiência de outros autores ${ }^{8}$.

Várias substâncias têm demonstrado possuir participação na gênese do dano cerebral. Estudos do metabolismo eicosanóide durante CEC, avaliando principalmente os níveis de tromboxano e prostacicli- nas, evidenciaram ações deletérias por parte destas substâncias sobre o sistema nervoso central 9,14 . Também foi evidenciado dano nas arteríolas cerebrais causado pelo ácido aracdônico e pela prostaglandina $\mathrm{G} 2{ }^{19}$. Recentemente, no estudo bioquímico e fisiopatológico dos processos que envolvem as membranas celulares durante hipoperfusão ou anoxia, foram descritos os radicais livres de oxigênio 11 .

Estas substâncias encontram-se muito aumentadas durante os procedimentos cirúrgicos cardíacos. DEMOPOULOS et alii ${ }^{7}$, em 1980 , publicaram extenso trabalho sobre a ação fisiopatológica dos radicais livres de oxigênio sobre a microcirculação cerebral. É importante ressaltarmos que as variações do $\mathrm{pH}$ e da $\mathrm{PaCO}_{2}$ foram por nós utilizadas como método indireto de avaliação da possibilidade de dano cerebral que pode ocorrer devido a alterações metabólicas durante este tipo de procedimento cirúrgico. Porém, não devemos esquecer que estas substâncias não são as únicas que podem estar envolvidas no dano cerebral.

O estudo experimental da hipoxia tecidual é muito complexo, requerendo dados algumas vezes difíceis de se obter como, por exemplo, a duração do tempo de apnéia, tempo de parada cardiaca, tempo de cianose ou hipotensão, monitorização contínua dos níveis pressóricos e alterações metabólicas locais, tais como acidose, hipercapnia, hipoglicemia e outras, de forma que - em nossa pesquisa - o número de variáveis foi muito grande. No entanto, foram tomados todos os cuidados para formar um grupo de estudo o mais homogêneo possível.

Em 1986, KATSUMIKO et alii ${ }^{18}$ investigaram a relação existente entre a velocidade de fluxo durante CEC e o consumo de oxigênio cerebral durante hipotermia profunda $\left(20^{\circ} \mathrm{C}\right)$ em cães, decrescendo a velocidade de fluxo de 100 para 60,30 e $15 \mathrm{ml} /$ $\mathrm{kg} / \mathrm{min}$. Neste trabalho, $60 \mathrm{ml} / \mathrm{kg} / \mathrm{min}$. parece ter sido o valor mais seguro na proteção do tecido cerebral, muito embora outros autores aceitem valores menores ${ }^{31}$. Em nossa pesquisa, $60 \mathrm{ml} / \mathrm{kg} / \mathrm{min}$. foi a velocidade de fluxo empregada durante o período de CEC e resfriamento sistêmico.

As estruturas cerebrais são perfundidas normalmente em proporção com a sua demanda metabólica ${ }^{40}$. A auto-regulação do fluxo cerebral ocorre em condições fisiológicas entre valores médios de $60 \mathrm{mmHg}$ a $150 \mathrm{mmHg}$ de pressão arterial, tanto em animais como no ser humano ${ }^{20}$. A redução da pressão arterial abaixo destes limites pode aumentar consideravelmente o risco de dano cerebral.

Alterações na pressão sistêmica durante a CEC são capazes de causar dano cerebral. WEJ et alii ${ }^{30}$, em 1980, constataram através de microscopia ele- 
ESPINOSA, G. L.; MURAD, H.; JAZBIK, A. P.; BASTOS, E. S.; BRITO, J. D.; FEITOSA, J. L.; BARROS, R. A. S.; D'ÁVILA, C. M. - Proteção cerebral no tratamento cirúrgico dos aneurismas do arco aórtico: estudo experimental em cães. Rev. Bras. Cir. Cardiovasc., 9 (4): 193-204, 1994.

trônica a presença de lesões vasculares na microcirculação cerebral, devido a um aumento da pressão arterial em gatos, submetidos experimentalmente a lesões traumáticas cerebrais. Em contrapartida, Roberts, em 1984, utilizando medidas espectrográficas das concentrações cerebrais de argônio, oxigênio e dióxido de carbono durante procedimentos cirúrgicos intracranianos, observou que a hipotensão intracraneana alterou importantemente o fluxo cerebral, a $\mathrm{PaO}_{2}$ e a $\mathrm{PaCO}_{2}$, locais. Em nosso trabalho, visando prevenir qualquer tipo de dano cerebral decorrente de alterações pressóricas sistêmicas durante a CEC., efetuou-se a monitorização contínua da pressão arterial média, que foi mantida em torno de $60 \mathrm{mmHg}$.

As técnicas de perfusão cerebral têm demonstrado resultados inconstantes na tentativa de conferir uma proteção cerebral satisfatória. Isto pode ser atribuído à instituição de um fluxo sangüíneo constante para o tecido cerebral, cuja necessidade pode variar ao longo da CEC, dependendo da atividade metabólica do cérebro. BLOODWELL et alii ${ }^{2}$ demonstraram o fenômeno do "furto da subclávia", quando apenas as carótidas são perfundidas durante a troca do arco aórtico.

Trabalhos onde a pressão sangüínea na artéria temporal foi monitorizada bilateralmente durante a CEC durante perfusão cerebral seletiva, têm demonstrado que valores acima de $40 \mathrm{mmHg}$ são satisfatórios na manutenção da auto-regulação cerebral ${ }^{29}$, muito embora autores prefiram valores um pouco mais elevados, em torno de $50-60 \mathrm{mmHg}$. Em nossa pesquisa, a pressão de perfusão carotídea foi mantida em torno de $60 \mathrm{mmHg}$, visando prevenir as alterações descritas.

Vários estudos indicam que, durante a CEC, ocorre a preservação da auto-regulação vascular cerebral, embora alguns trabalhos colocam esse fato em dúvida. A maioria das pesquisas sugere que se deve tentar manter a auto-regulação cerebral, a fim de prevenir possíveis alterações neurológicas especialmente quando for utilizada a perfusão cerebral seletiva na abordagem cirúrgica do arco aórtico 2 .

Estudos sobre o fluxo cerebral e a função microvascular têm demonstrado que, quando associamos a CEC com a hipotermia profunda, ocorre uma perda da auto-regulação da microcirculação cerebral ${ }^{15}$, possuindo o córtex cerebral, cerebelo e gânglios da base uma tendência a lesões hiperêmicas e a substância branca cerebral, a lesōes isquêmicas ${ }^{22}$. Isto justifica a grande congestão vascular cortical encontrada nos animais estudados nesta pesquisa, que foi sensivelmente maior nos animais submetidos a pinçamento arterial. A perfusão carotídea, embora tenha minimizado este efeito, não proporcionou o aparecimento de uma congestão vascular cortical leve.
Os efeitos da parada cardiocirculatória total podem se manifestar não só sobre os centros nervosos superiores, como também sobre a medula espinhal. Em nossa pesquisa, a CEC foi mantida durante o período de hipotermia e pinçamento arterial dos vasos do arco aórtico, no sentido de proteger não só a medula espinhal, como os demais tecidos nobres. A manutenção da CEC neste período proporciona excelente via arterial para a colocação de cânula na carótida.

Episódios de acidose em hipoxia, diminuição da $\mathrm{PaCO}_{2}$ podem acarretar o edema tecidual, pois a isquemia produz alterações na barreira hematencefálica. Estas alterações são fundamentais para que ocorra o edema cerebral, pois apenas a necrose celular neuronal é insuficiente. A lesão da substância branca pode ocorrer em situações mais severas.

Em condições experimentais, o neurônio é a primeira estrutura a ser lesada. A anoxia neuronal clássica, vista em cérebros maduros, caracteriza-se por marcante eosinofilia do citoplasma, perda da substância de Nissl e picnose nuclear. Estas alterações podem surgir em condições normotérmicas a partir de cinco minutos de hipoxia. Em nossa pesquisa, este tipo de lesão celular, compatível com isquemia neuronal, foi amplamente observado, principalmente nos animais onde efetuou-se apenas pinçamento arterial. A intensidade destas lesões tornou-se mais exuberante quanto maior o tempo de pinçamento arterial.

A perfusão carotídea minimizou em muito o grau de lesão isquêmica neuronal, porém não foi capaz de abolir completamente, sendo encontradas as lesões mesmo nos animais submetidos ao menor tempo do procedimento (45 $\mathrm{min}$.).

Normalmente, é necessário que decorram 24 horas para que as lesões isquêmicas se tornem bem evidentes em toda sua magnitude à microscopia óptica, muito embora possa ser observado dano cerebral com menos de 6 horas do evento isquêmico ${ }^{13}$. Alterações com pouco tempo de evolução podem ou não ser vistas ao microscópio óptico, o que torna difícil estabelecer um padrão de lesão pelo exame deste tipo de tecido cerebral.

Pelo exposto, é de supor que as lesões isquêmicas encontradas nesta pesquisa são representativas de lesões isquêmicas mais importantes já que - tempo decorrido entre o episódio isquêmico e a retirada da massa encefálica foi inferior a 24 horas.

Segundo a literatura, a distribuição dos locais de lesão devida à hipoxia ocorre em ordem decrescente de frequeência: no tálamo, gânglios da base, córtex e região pontino-bulbar ${ }^{24}$. Estes locais podem ser acometidos isoladamente ou em associação. 
ESPINOSA, G. L.; MURAD, H.; JAZBIK, A. P.; BASTOS, E. S.; BRITO, J. D.; FEITOSA, J. L.; BARROS, R. A. S.; D'ÁVILA, C. M. - Proteção cerebral no tratamento cirúrgico dos aneurismas do arco aórtico: estudo experimental em cães. Rev. Bras. Cir. Cardiovasc., 9 (4): 193-204, 1994.

Em nossa pesquisa, esta ordem de incidência não foi confirmada, sendo o córtex a área mais lesada, principalmente na região fontal, que apresentava algum grau de comprometimento praticamente em todos os animais. Estes achados podem ser conseqũentes ao fato do cão apresentar, devido ao bulbo olfatório, um córtex frontal desenvolvido.

Alteraçōes isquêmicas podem ser comumente encontradas em pequena quantidade em tecidos cerebrais normais, como conseqüência do método utilizado para a fixação tecidual. Este fato explica as alterações isquêmicas mínimas encontradas em alguns animais do grupo controle, e que carecem de maior relevância.

A hipoxia predispõe à hemorragia tecidual. A reperfusão de uma área isquêmica pode acarretar a hemorragia cerebral parenquimatosa ou subcortical, principalmente quando associada a um aumento da pressão capilar ou venosa.

Devemos diferenciar a hemorragia primária do tecido cerebral da hemorragia secundária a áreas de isquemia ou necrose.

Em nossa pesquisa, foram observados alguns casos de hemorragia nos animais submetidos a perfusão carotídea seletiva. Isto deveu-se provavelmente a manutenção de uma pressão sangüinea capilar elevada, graças à presença de um fluxo constante, durante o período de hipoxia tecidual. Talvez uma velocidade de fluxo menor possa diminuir a incidência deste tipo de complicação, abrindo um campo de estudo de interesse para futuras investigações.

O uso de perfusão cerebral retrógrada defendido por YASUURA et alii ${ }^{32}$ pode, talvez, oferecer proteção cerebral à isquêmia mais prolongada. Este é um tema que necessita ser estudado com maior profundidade.

\section{CONCLUSÕES}

Aos 45 minutos de isquemia cerebral, ambos os métodos estudados nesta pesquisa conferiram relativa proteção cerebral, com evidência histopatológica de discretas lesões isquêmicas. Não ocorreram alterações metabólicas.

Aos 90 minutos de isquemia cerebral, houve melhor proteção cerebral nos animais do Grupo II (com cânula na carótida); o número de lesões isquêmicas celulares neuronais, bem como as alteraçōes metabólicas, foram consideravelmente menores.

Aos 135 minutos de isquemia cerebral, não houve superioridade de um método sobre o outro; ambos foram incapazes de conferir proteção cerebral. O Grupo II apresentou menores alteraçōes metabólicas.

O tempo de isquemia cerebral ainda é fator limitante. Esta pesquisa mostrou que este tempo pode ser aumentado para 90 minutos, através do uso da perfusão carotídea seletiva.

ESPINOSA, G. L.; MURAD, H.; JAZBIK, A. P.; BASTOS, E. S.; BRITO, J. D.; FEITOSA, J. L.; BARROS, R. A. S.; D'ÁVILA, C. M. - Cerebral protection during surgical approach to the aortic arch aneurysms resection: experimental study in dogs. Rev. Bras. Cir. Cardiovasc., 9 (4): 193-204, 1994.

ABSTRACT: The authors proposition is to make an experimental study of two methods of cerebral protection to be used during aortic arch aneurysm resection. The methods to be evaluated were profound systemic hypothermia (under $20^{\circ} \mathrm{C}$ ) whith great vessels occlusion and profound systemic hypothermia with selective right carotid artery perfusion. Two groups of 15 dogs each were submitted either to profound systemic hypothermia with great vessels occlusion (Group I), or to profound systemic hypothermia with selective right carotid artery perfusion (Group II). Serial jugular vein samples for $\mathrm{pH}$ and $\mathrm{PaCO} 2$ were analyzed to evaluate ischemic cerebral metabolic derangements. Hystopathological studies were also performed 45,90 and 135 minutes, through animal sacrifice in each experiment. The results have shown good cerebral protection with both methods in the 45 minutes ischemic interval. At 90 minutes Group II method has conferred better cerebral protection than Group I. At 135 minutes of cerebral ischemia neither method could afford cerebral protection against ischemia.

DESCRIPTORS: cerebral protection; aortic arch aneurysms, surgery. 
ESPINOSA, G. L.; MURAD, H.; JAZBIK, A. P.; BASTOS, E. S.; BRITO, J. D.; FEITOSA, J. L.; BARROS, R. A. S.; D'ÁVILA, C. M. - Proteção cerebral no tratamento cirúrgico dos aneurismas do arco aórtico: estudo experimental em cães. Rev. Bras. Cir. Cardiovasc., 9 (4): 193-204, 1994.

\section{REFERÊNCIAS BIBLIOGRÁFICAS}

1 BACHET, J.; TEODORI, G.; GOUDOT, B.; DIAZ, F.; KERDANY, A.; DUBOIS, C.; BRODATY, D.; DE LENTDEKER, P.; GUILMET, D. - Replacement of the transverse aortic arch during emergency operations for type A acute aortic dissection. J. Thorac. Cardiovasc. Surg., 96: 878-886, 1988.

2 BLOODWELL, R. D.; HALLMAN, G. L.; COOLEY, D. A. - Total replacement of the aortic arch and the "Subclavian Steal" phenomenon. Ann. Thorc. Surg., 5: 236-245, 1968.

3 COOLEY, D. A.; OTT, D. A.; FRAZIER, O. H.; WALKER, W. E. - Surgical treatment of aneurysms of the transverse aortic arch: experience with 25 patients using hypothermic techniques. Ann. Thorac. Surg., 32: $260-272,1981$.

4 CRAWFORD, E. S. \& SALEH, S. A. - Transverse aortic arch aneurysm: improved results of treatment employng new modifications of aortic reconstruction and hypothermic cerebral circulatory arrest. Ann. Surg., 194: 180-188, 1981.

5 DE BAKEY, M. E. - Succesful resection of fusiform aneurysm of aortic arch with replacement by homograft. Surg. Gynecol. Obstet., 105: 657-664, 1957.

6 DE BAKEY, M. E.; CRAWFORD, E. S.; COOLEY, D. A. - Successful resection of fusiform aneurysm of aortic arch with replacement by homograft. Surg. Gynecol. Obstet., 105: 657-664, 1957.

7 DEMOPOULOS, H. B.; FLAMM, E. S.; PIETRONIGRO, D. D.; SELIGMAN, M. L. - The free radical pathology and the microcirculation in the major central nervous system. Acta Physiol. Scand., 91: (Supl. 492): 119, 1980.

8 EKROTH, R.; THOMPSON, R. J.; LINCOLN, C.; SCALLAM, M.; ROSSI, R.; TSANG, V. - Elective deep hypothermia with total circulatory arrest: changes in plasma creatine kinase BB, blood glucose, and clinical variables. J. Thorac. Cardiovasc. Surg., 97: 30-35, 1989.

9 FISH, K. J.; HELMS, K. N.; SARQUIST, F. H.; VAN STIEENNIS, C.; LINET, O. I.; HILBERMAN, M.; MITCHELL, R. S.; JAMIELSON, S. W.; MILLER, D. C.; TINCLENBERG, J. S. - A prospective, randomized study of the effects of prostacyclin on neuropsychologic dysfuction after coronary artery operation. J. Thorac. Cardiovasc. Surg., 93: 605-615, 1987.

10 FOX, L. S.; BLACKSTONE, E. H.; KIRKLIN, J. W.; BISHOP, S. P.; BERGDAHL, L. A.; BRADLEY, E. L. - Relationship of brain blood flow and oxygen consumption to perfusion flow rate during profoundly hypothermic cardiopulmonary bypass. J. Thorac. Cardiovasc. Surg., 87: 658-664, 1984.

11 FRIDOVICH, I. - The biology of oxygen radicals; the superoxide radical is an agent of oxygen toxicity; superoxide dismutases provide an important defense. Science, 201: 875-880, 1978.

12 FRIST, E. H.; BALDWIN, J. C.; STARNES, V. A.; DYER, P. E.; MILLER, D. C.; JAMIESON, S. W.; MITCHELL, R. S.; SHUMWAY, N. E. - A reconsideration of cerebral perfusion in aortic arch replacement. Ann. Thorac. Surg., 42: 273-281, 1986.

13 GARCIA, J. H. \& KAMIJYO, Y. - Cerebral infarction: evolution of histopathological changes after occlusion of a middle cerebral artery in primates. J. Neuropathol. Exp. Neurol., 33: 408-421, 1974.

14 GREELEY, W. J.; BUSHMAN, G. A.; KONG, D. L.; OLDHAM, H. N.; PETERSON, M. B. - Effects of cardiopulmonary bypass on metabolism during pediatric cardiovascular surgery. J. Thorac. Cardiovasc. Surg., 95: 842-849, 1988.

15 GREELEY, W. J.; UNGERLEIDER, R. M.; SMITH, R. L.; REVES, J. G. - The effects of deep hypothermic cardiopulmonary bypass and total circulatory arrest on cerebral blood flow in infants and children. $J$. Thorac. Cardiovasc. Surg., 97: 737-745, 1989.

GRIEPP, R. B.; STINSON, E. B.; HOLLINGSWORTH, J. F.; BUEHLER, G. - Prosthetic replacement of the aortic arch. J. Thorac. Cardiovasc. Surg., 70: 1051$1063,1975$.

KAMIJYO, Y.; GARCIA, J. H.; COOPER, J. - Temporary regional cerebral ischemia in the cat: a model of hemorrhagic and subcortical infarction. J. Neuropathol. Exp. Neutrol., 36: 338-350, 1977.

KATISUHIKO, M.; YASUNARU, K.; MATSUDA, H.; OKUDA, A.; ELLIS, E. F. - Optimal perfusion flow rate for the brain during deep hypothermic cardiopulmonary bypass at $20^{\circ} \mathrm{C}$ : an experimental study. $\mathrm{J}$. Thorac. Cardiovasc. Surg., 92: 1065-1070, 1986.

KONTOS, H. A.; WEI, E. P.; POVLISHOCK, J. T.; DIETRICH, W. D.; MAGIERA, G. T.; ELLIS, E. F. Cerebral arteriolar dammage by arachidonic acid and prostaglandin G2. Science, 209: 1242-1245, 1980.

20 LASSEN, N. A.; Cerebral blood flow and oxygen consumption in man. Physiol. Rev., 39: 183-238, 1959.

21 LUOSTO, R.; MAAMIES, T.; PELTOLA, K.; JARVINEN, A.; MATTILA, S. - Hypothermia and circulatory arrest in reconstruction of aortic arch: a report of nine cases. Scand. J. Thorac. Cardiovasc. Surg., 21: 113-117, 1987.

22 MINORU, A.; FLASTERSTEIN, A. H.; BARNETTE, J.; $\mathrm{KOCH}$, L. V.; ROSS Jr., J. N.; KENNEDY, J. H. Cerebral effects of profound hypothermia $\left(18^{\circ} \mathrm{C}\right)$ and circulatory arrest. Circulation, 51, 52 (Supl. 1): 52 $59,1975$. 
ESPINOSA, G. L.; MURAD, H.; JAZBIK, A. P.; BASTOS, E. S.; BRITO, J. D.; FEITOSA, J. L.; BARROS, R. A. S.; D'ÁVILA, C. M. - Proteção cerebral no tratamento cirúrgico dos aneurismas do arco aórtico: estudo experimental em cães. Rev. Bras. Cir. Cardiovasc., 9 (4): 193-204, 1994.

23

PHILIPS, P. A. \& MIYAMOTO, A. M. - Use of hypothermic and cardiopulmonary bypass in resection of aortic arch aneurysms. Ann. Thorac. Surg., 17: 398-404, 1974.

RANCK, J. B. \& WINDLE, W. F. - Brain damage in the monkey, maccaca mulatta, by asphyxia neonatorum. Exp. Neurol. 1: 130, 1959.

SHU-HSUN, C.; YUAN-TEH, L.; WEN-PIN, L.; CHU, S. H.; LEE, Y. T.; LIEN, W. P.; HSU, K. Y.; WU, T. L. - Resection of aneurysm of the aortic arch without cardiopulmonary bypass. J. Thorac. Cardiovasc. Surg., 74: 928-934, 1977.

SWAN, H. - The importance of acid-base management for cardiac and cerebral preservation during open heart operations. Surg. Gynecol. Obstet., 158: 391 . 414, 1984.

27 TABADOR, K.; GARDNER, T. J.; WALKER, A. E. Cerebral circulation and metabolism at deep hypothermia. Neurology, 22: 1065-1070, 1972.

TANAKA, J.; SHIKI, K.; ASOU, T.; YASUI, H.; TOKUNAGA, K. - Cerebral autoregulation during deep hypothermic nonpulsatile cardiopulmonary bypass with selective cerebral perfusion in dogs. J. Thorac. Cardiovasc. Surg., 95: 124-132, 1988.

29 TREASURE, T.; NAFTEL, D. C.; CONGER, K. A.; GARCIA, KIKLIN, J. W.; BLACKSTONE, E. H. - The effect of hypothermic circulatory arrest time on cerebral function, morphology, and biochemistry: an experimental study. J. Thorac. Cardiovasc. Surg., 86: 761-770, 1983.

30 WEI, E. P.; DIETRICH, D.; POVLISHOCK, T. J.; NAVARI, R. M.; KONTOS, H. A. - Functional, morphological and metabolic anormalities of the cerebral microcirculation after concussive brain injury in cats. Circulation Res., 46: 37-47, 1980.

31 YASUHIRO, S.; TAKASHI, M.; RYOHEI, Y.; SOMA, Y.; HIROTANI, T.; YOZU, R.; ONOGUSHI, K.; MISUMI, T.; KAWADA, K.; INOUE, T. - A clinical study of cerebral circulation during extracorporeal circulation. J. Thorac. Cardiovasc. Surg., 97: 187-193, 1989.

32 YASUURA, K.; OGAWA, Y.; OKAMOTO, H. S.; ASAKURA, T.; OSHINO, M.; SAWAZAKI, M.; MATSURA, A.; MASEKI, T.; ABE, T. - Clinical application of total body retrograde perfusion to operation for aortic dissection. Ann. Thorac. Surg., 53: 655-660, 1992. 